\title{
Order in Chaotic Oscillation in Nonlinear Systems
}

\author{
Hirokazu Ohtagaki and Takahiro Yamaguchi \\ Dept. of Electrical and Electronics Engineering, Okayama University of Science \\ 1-1 Ridai-cho, Kita-ku, Okayama 700-0005, Japan \\ E-mail: ohtagaki@ee.ous.ac.jp
}

\begin{abstract}
This paper proposes a method invoking one dimensional piecewise continuous map(PCM) in order to investigate the order in chaotic oscillation and periodic oscillation in nonlinear dynamical systems described by ordinary differential equations. The systems studied in this paper are of Van der Pol-Mathieu type.

The PCM is derived from the data sequence of state variables of nonlinear systems. Computational experiments on the systems of Van der Pol-Mathieu type and on the PCM show that the proposed method is effective to investigate the existence of chaotic oscillation and periodic oscillation in nonlinear dynamical systems such as Van der Pol-Mathieu type.
\end{abstract}

\section{Introduction}

Since 1976, chaotic phenomena are studied in many published papers [1], [2]. The importance of nonlinear dynamics has been considerably intensified by researchers $[3] \sim[5]$. Xin et al. and Liu et al. presented the chaos synchronization method to synchronize two identical chaotic systems with different initial values [4] $\sim[6]$.

Studies of nonlinear systems of Van der Pol type are presented in the papers [7] [12]. In the paper [13], only a sine circle map to the nonlinear systems of van der Pol type is shown. The paper [13] can not show the efficiency of the sine circle maps.

In this paper, a method invoking a one dimensional piecewise continuous map(PCM) is proposed to investigate the existence of chaotic oscillation and periodic oscillation in nonlinear dynamical systems described by ordinary differential equations. The systems studied in this paper are of Van der Pol-Mathieu type.

At first, a sine circle map is derived from data sequence of the state variables of the system, and then a PCM is derived to approximate the sine circle map.

Secondly, a rotation number and a Lyapunov exponent in the PCM are introduced to investigate the mapped points in the PCM.

Finally, from computational experiments on the results obtained from systems of Van der Pol-Mathieu type and on the results obtained from the PCM, it is shown that the proposed method is effective to investigate the existence of chaotic oscillation and periodic oscillation in nonlinear dynamical systems such as Van der Pol-Mathieu type.

The paper is organized as follows. In section 2 , a sine circle map and an approximate PCM are derived. In section 3 , it is shown that rotation numbers in the PCM form a subset of Farey series. In section 4, computational experiments are presented to show that the proposed method is effective to investigate the existence of chaotic oscillation and periodic oscillation in nonlinear dynamical systems such as Van der Pol-Mathieu type. In section 5 , concluding remarks of this paper are presented.

\section{Sine Circle Map and Piecewise Continuous Map}

Dynamics of the nonlinear system of Van der PolMathieu type is written by the ordinary differential equation;

$$
\ddot{x}+\left(\beta-\delta x^{2}\right) \dot{x}+\left(1+\gamma x^{2}+\mu \cos (\omega \mathrm{t})\right) x=0,
$$

where $\beta$ and $\delta$ are damping constants, $\gamma$ is a nonlinear parameter, $\mu$ and $\omega$ are an amplitude and an angular frequency of an internal excitation, respectively. It is shown that the dynamical behavior in this nonlinear system exhibits a chaotic motion in certain region of damping coefficients (see [17]).

An illustrative oscillation in the state variable $\boldsymbol{x}=$ $\left(x_{1}, x_{2}\right)^{\prime}$ is shown in Fig.1(a), (b) and its phase plane portrait is shown in Fig.1(c).

The angle variables to the state variables are defined as

$$
\theta_{n}=\tan ^{-1}\left(\frac{x(n T)}{\dot{x}(n T)}\right), \quad n=0,1, \ldots
$$

where $T(=2 \pi / \omega)$ is a period of internal excitation (see Fig.2). From the data sequence $\{\boldsymbol{x}(n T)(=$ $\left.\left.\left.(x(n T), \dot{x}(n T))^{\prime}\right)\right) \mid n=1,2, \ldots\right\}$ in the system equation (1), a sine circle map is expressed by a map of dimension one;

$$
\theta_{n+1}=f\left(\theta_{n}\right), \quad n=0,1, \ldots
$$

where the sequence of the angle variables are obtained from the data $\{\boldsymbol{x}(n T) \mid n=1,2, \ldots\}$ (see Figs.3 and 4). 


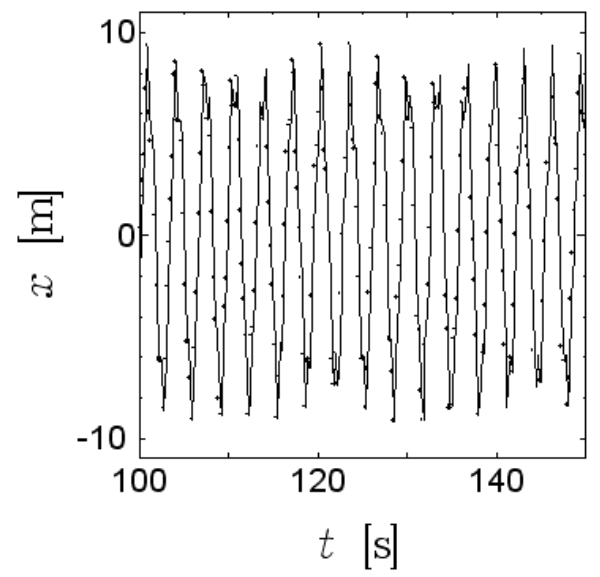

(a)

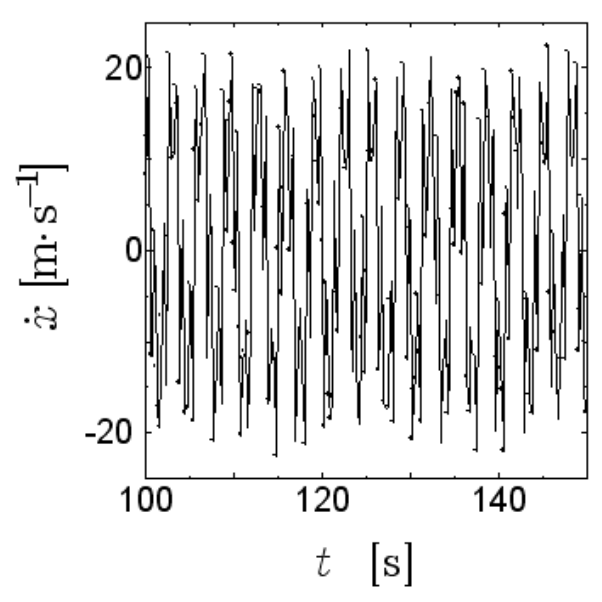

(b)

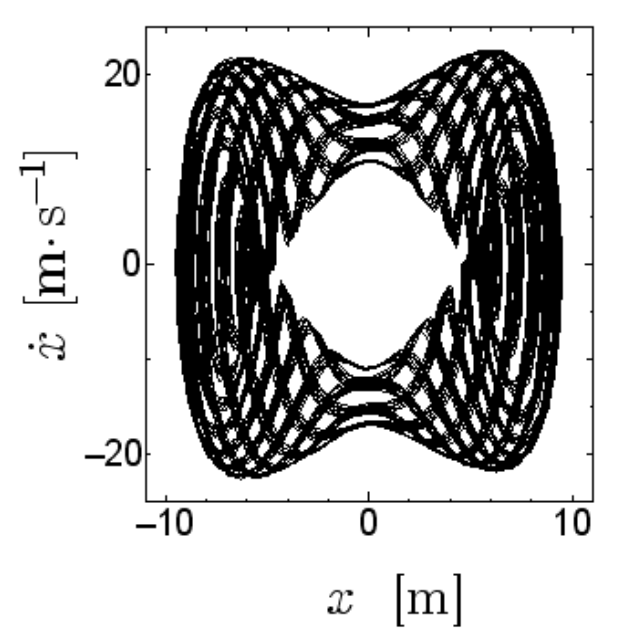

(c)

Fig. 1: Oscillation in Nonlinear Systems of Van der Pol-Mathieu Type $(\beta=0.054$, $\delta=0.01, \gamma=0.01, \mu=20.0, \omega=10.0)$

By using root mean square method to the data sequence of sine circle map, an approximate piecewise continuous map (PCM) to the sine circle map $f$ can be expressed as

$$
\begin{gathered}
\theta_{n+1}=f_{1}\left(\theta_{n}\right):=A\left(\theta_{n}+C\right), \\
\left(0 \leq \theta_{n}<\tilde{D}(=1 / A-C)\right), \\
\theta_{n+1}=f_{2}\left(\theta_{n}\right):=B\left(\theta_{n}-\tilde{D}\right), \\
\left(\tilde{D} \leq \theta_{n} \leq 1\right) .
\end{gathered}
$$

where angle variable $\theta_{n}$ is normalized as $0 \leq \theta_{n} \leq 1, A$ and $B$ are gradients of the maps $f_{1}$ and $f_{2}$, respectively, and $\tilde{D}$ is a cut-off parameter of the PCM.

The sine circle map and its approximate PCM are shown in Fig. 4 where the curves are of sine circle map and straight lines are of the approximate PCM. From Fig.4, it can be seen that the sine circle map is well approximated by the PCM.

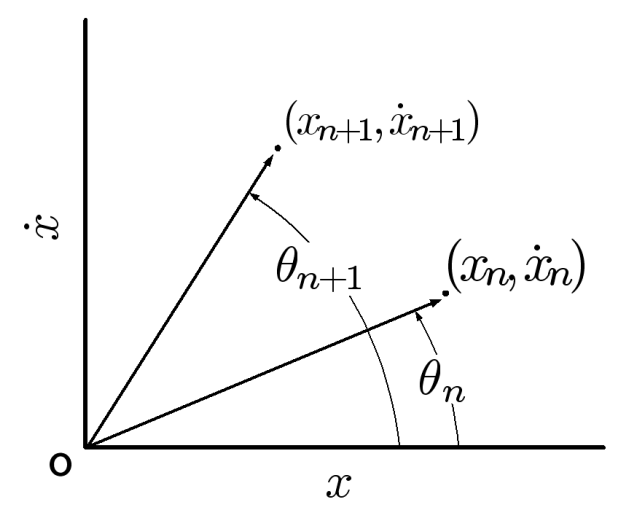

Fig. 2: Angle Variables and State Variables

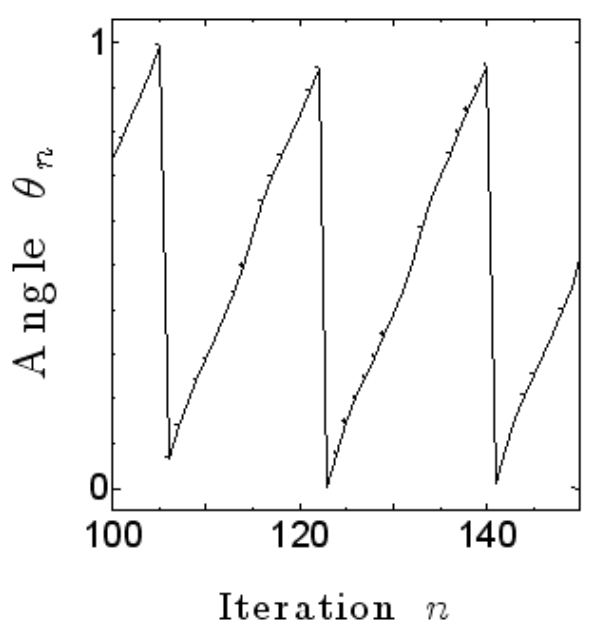

Fig. 3: Data Sequence of Angle Variables

\section{Rotation Number and Lyapunov Exponent}

In this section, rotation numbers and Lyapunov exponents are introduced to investigate motions in the PCM. 


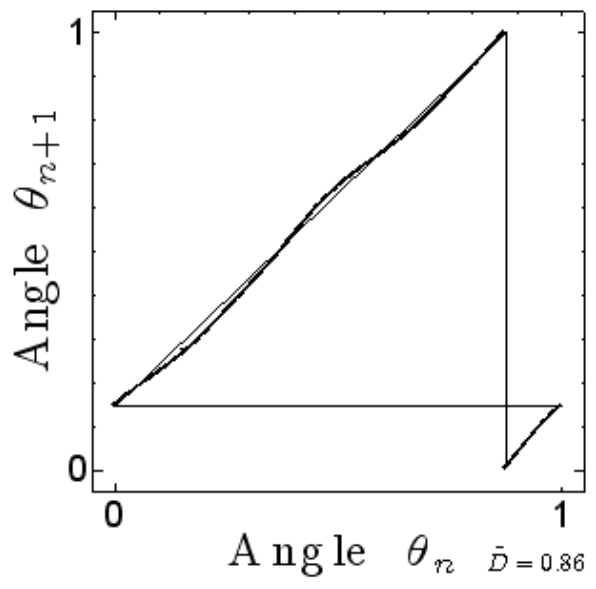

Fig. 4: Sine Circle Map and its Approximate PCM $\quad(A=0.9, B=1.1)$

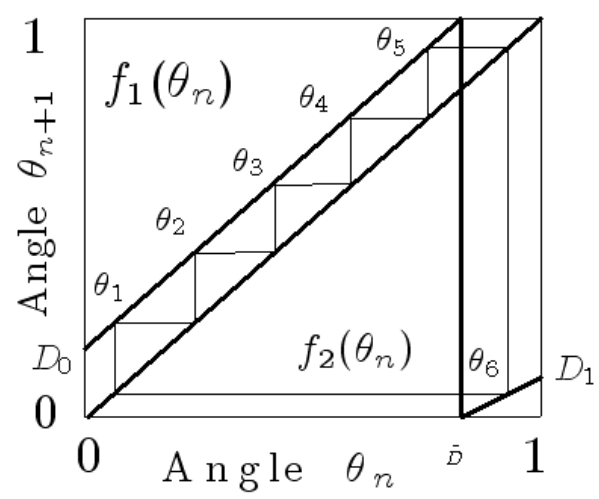

Fig. 5: Motion of Mapped Points in the PCM $\left(A=0.9, B=1.1, D_{0}=0146, D_{1}=0.159\right)$

Let the periodic motion of period $m$ in the PCM be denoted by $\pi(m)$. In Fig.5, the motion of $\pi(6)$ is shown, where $\theta_{1}, \theta_{2}, \ldots, \theta_{6}$ indicate mapped points in the PCM. A rotation number to the PCM is defined by

$$
W=\frac{1}{m} .
$$

Eq. (4) means that winding motion of one is generated by $f_{2} \circ f_{1}^{m-1}$, where $m=6$ in the motion shown in Fig.5.

In the PCM, a motion of $r$ times $m$ peaks $(r \cdot m)$ and $s$ times $(m+1)$ peaks $(s \cdot(m+1))$ is denoted as $\pi_{r, s}(m, m+1)$, where $r, s$ and $m$ are positive integers. Similarly, quasi-periodic motion of $r$ times $m$ peaks $s$ times $m+1$ peaks is denoted as $\tilde{\pi}_{r, s}(m, m+1)$.

Mixing degree $P$ and multiplicity $M$ in the motion $\pi_{r, s}(m, m+1)$ and $\tilde{\pi}_{r, s}(m, m+1)$ are defined as follows;

$$
\begin{aligned}
P & =r+s, \\
M & =m \cdot r+(m+1) \cdot s(=m \cdot P+s) .
\end{aligned}
$$

Rotation numbers $W$ of the motions $\pi_{r, s}(m, m+1)$ and $\tilde{\pi}_{r, s}(m, m+1)$ in the PCM can be defined as

$$
W=\frac{P}{M}=\frac{r+s}{r \cdot m+s \cdot(m+1)},
$$

which means that $r+s$ windings are generated by $r$. $m+s \cdot(m+1)$ iterations of mappings in the PCM.

Lyapunov exponents of mapped points with $Q$ points generated by $f_{1}$ and $R$ points generated by $f_{2}$ in the $\mathrm{PCM}$ can be defined as

$$
L=\frac{1}{Q+R} \log \left(A^{Q} \cdot B^{R}\right) .
$$

Eq.(7) is rewritten as

$$
\begin{aligned}
L=\frac{1}{Q+R} & \left((Q+R) \log B\left(1-\frac{\frac{Q}{Q+R}}{\frac{\log B}{\log A}}\right)-Q \log B\right) \\
& =\left(1-\frac{\frac{Q}{Q+R}}{\frac{\log B}{\log A}}\right)\left(\frac{Q}{Q+R}\right) \log B .
\end{aligned}
$$

It is well known that if the Lyapunov exponent $L>0$, then the motions of the mapped points are chaotic and that if the Lyapunov exponent $L<0$, then the motions of the mapped points are periodic.

Let the value of the cut-off parameter $\tilde{D}$ such that $L=0$ be $\tilde{D}_{\mathrm{C}}$, which is called a critical cut-off parameter.

From the results of computational experiments shown in Fig.6, the following proposition is stated.

Proposition 1 (Existence of Chaotic Motion and Existence of Periodic Motion)

If, for the cut-off parameter $\tilde{D}$, an inequality

$$
\tilde{D}>\tilde{D}_{\mathrm{C}}
$$

holds, then the motion in the PCM is chaotic or quasiperiodic.

If, for the cut-off parameter $\tilde{D}$, an inequality

$$
\tilde{D}_{\mathrm{C}}>\tilde{D}
$$

holds, then the motion in the PCM is periodic.

Equation (8) can be rewritten as:

$$
L=\frac{1}{Q+R}\left(\log A^{Q}+\log B^{R}\right) .
$$

In the Eq.(10), let $Q=1, R=m_{0}$ be the numbers satisfies equality $L=0$. From Eq.(10), it follows that

$$
\begin{gathered}
L=\frac{1}{m_{0}}\left(\left(m_{0}-1\right) \log B+\log A\right)=0, \\
m_{0}=1-\frac{\log A}{\log B} .
\end{gathered}
$$

Therefore, from Eq.(12), it follows that the Corollary 1 holds for $0<A<1<B$. 


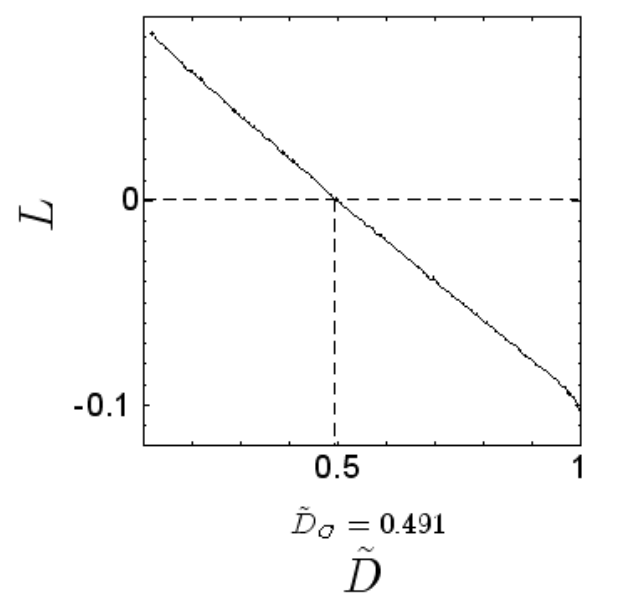

Fig. 6: Lyapunov Exponent in PCM

\section{Corollary 1 (Existence of Periodic Motion of} $\pi(m))$

There exist periodic motions $\pi(m)$ for positive integer $m$ that satisfies the inequality

$$
m \geq M_{\min }=\left[m_{0}\right],
$$

where $[\cdot]$ denotes a least integer greater than the real number $(\cdot)$.

From Corollary 1 and Eq.(12), it follows that the periodic motions $\pi(m)$ exist for $m$ such that

$$
m \geq M_{\min }=2\left(>m_{0}=1.905\right)
$$

for $A=0.9$ and $B=1.1$, which are derived from the sine circle map associated with the van der PolMathieu system $(\beta=0.055$, see Figs. 7 and 8$)$. Figure 7 shows the dependencies of $A, B$ and $L(\beta)$ on the damping parameter $\beta$. In Fig. $8, L_{M}(\beta)$ denotes maximum Lyapunov exponents of Van der Pol-Mathieu systems and $L(\beta)$ denotes Lyapunov exponents of the PCM. From Figs. 7 and 8 , it is seen that if $L(\beta)>0$, then $L_{M}(\beta)>0$ at almost every where, therefore Lyapunov exponents of the PCM are effective for detecting chaotic oscillations in the Van der Pol-Mathieu systems.

\section{Computational Experiments}

In this section, results on computational experiments are shown to effectiveness of the method proposed in this paper.

Bifurcation diagrams associated with the PCM are shown in Fig.9. Various motions of $\tilde{\pi}_{r, 1}(m, m+1)$ and $\tilde{\pi}_{1, s}(m, m+1)$ are shown in Fig.9 for certain regions of cut-off parameter. It is shown that motions exist successively $\tilde{\pi}_{1,3}(4,5), \tilde{\pi}_{1,2}(4,5), \tilde{\pi}_{1,1}(4,5), \tilde{\pi}_{2,1}(4,5)$, $\tilde{\pi}_{3,1}(4,5)$, which correspond to rotation numbers in series;

$$
\{4 / 19,3 / 14,2 / 9,3 / 13,4 / 17\}
$$

and form a part of a successive Farey series with denominator less than 19. The Farey series $F_{M}$ of order $M$ is the monotonically increasing sequence of all irreducible rationales between 0 and 1 whose denominators do not exceed $M$, thus $\frac{P_{i}}{M_{i}}$ belongs

$$
F_{M}=\left\{\frac{1}{5}, \frac{1}{4}, \frac{1}{3}, \frac{2}{5}, \frac{1}{2}, \frac{3}{5}, \frac{2}{3}, \frac{3}{4}, \frac{4}{5}, \frac{1}{1}\right\} .
$$

A Farey sequence has a property such that if $\frac{P_{i-1}}{M_{i-1}}$ and $\frac{P_{i}}{M_{i}}$ are consecutive terms of $F_{M}$, then

$$
P_{i} M_{i-1}-P_{i-1} M_{i}=1 .
$$

From Fig.9, it can be seen that the motions in the PCM is of period $m \geq 2$ exist for $\tilde{D}>\tilde{D}_{\mathrm{C}}$.

For the value of damping parameter $\beta=0.055$, the values in approximate PCM are $A=0.9, B=1.1$ and $\tilde{D}=0.86$. From Figs.6,8 and 9 , it can be seen that the motions in the PCM are chaotic and the oscillations in the nonlinear systems of Van der Pol-Mathieu type are also chaotic.

In Figs.10 and 11, the motions in the PCM are shown to $\pi_{1,1}(4,5)$ and $\pi(2)$. The motion in Fig. 11 is of almost periodic. The motion in Fig. 12 is of quasi-periodic.

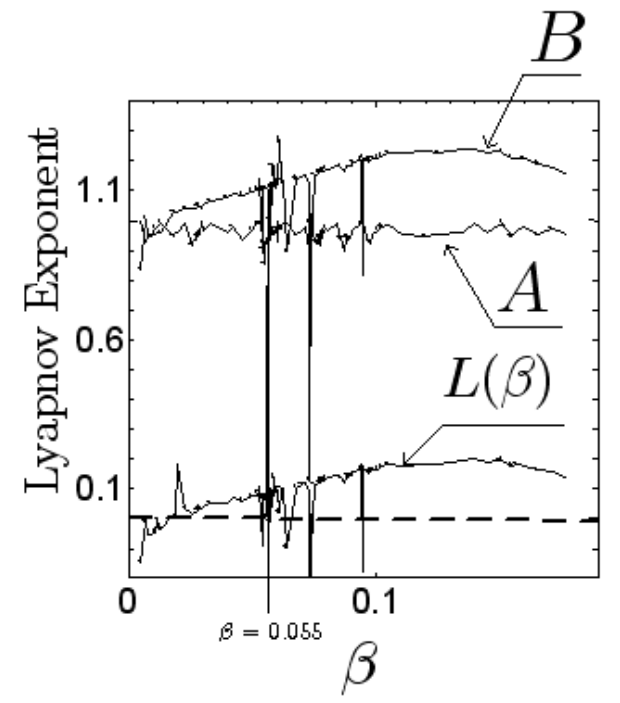

Fig. 7: Lyapunov Exponents of Mapped Points in the PCM

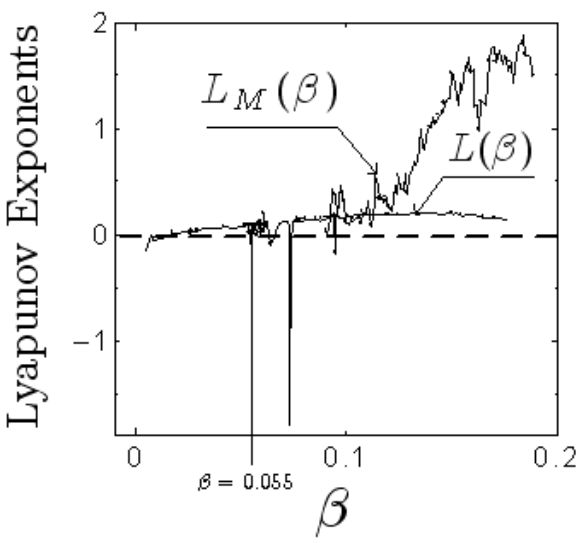

Fig. 8: Lyapunov Exponents of Mapped Points in the PCM and Nonlinear Systems of Van der Pol-Mathieu Type 


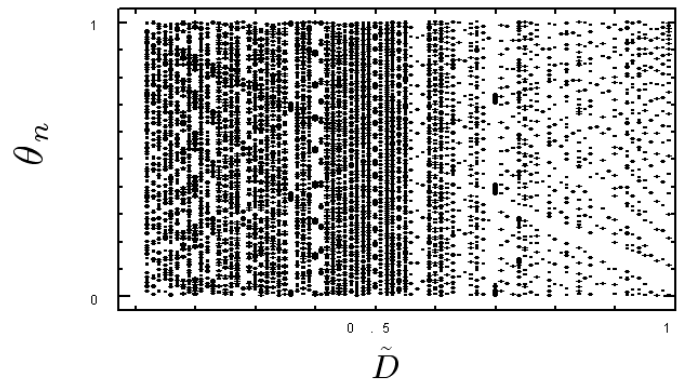

(a)

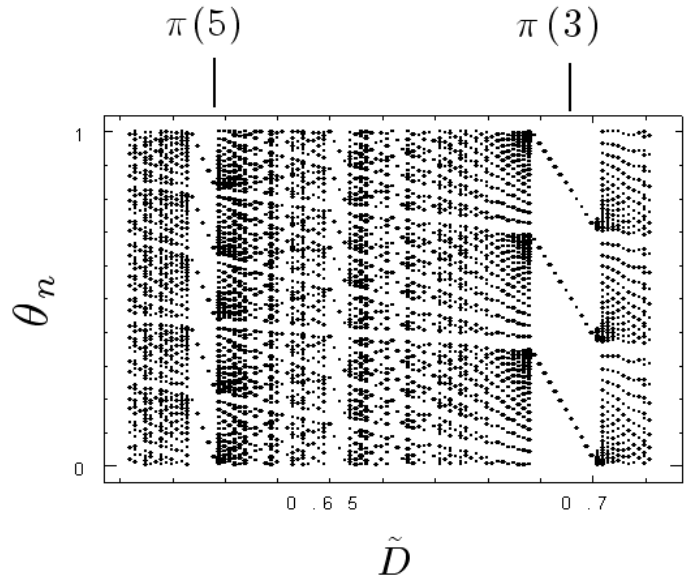

(b)

Fig. 9: Bifurcation Diagram of the mapped points in PCM

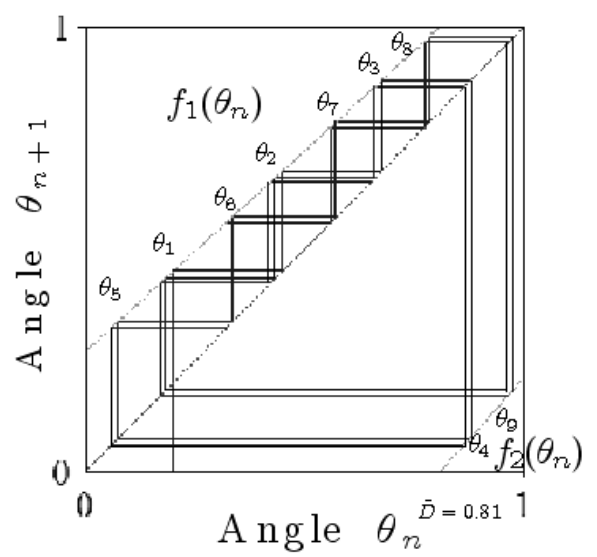

Fig. 10: Motion in PCM $\left(\pi_{1,1}(4,5)(\tilde{D}=0.81)\right)$

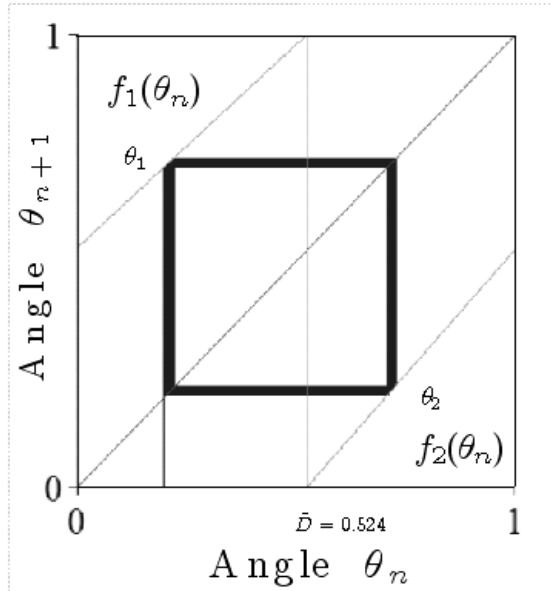

Fig. 11: Motion in PCM $(\pi(2))(\tilde{D}=0.524)$

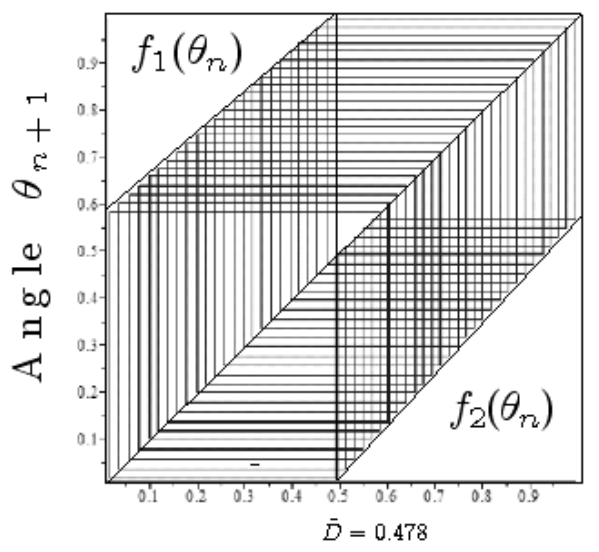

Angle $\theta_{n}$

(a)

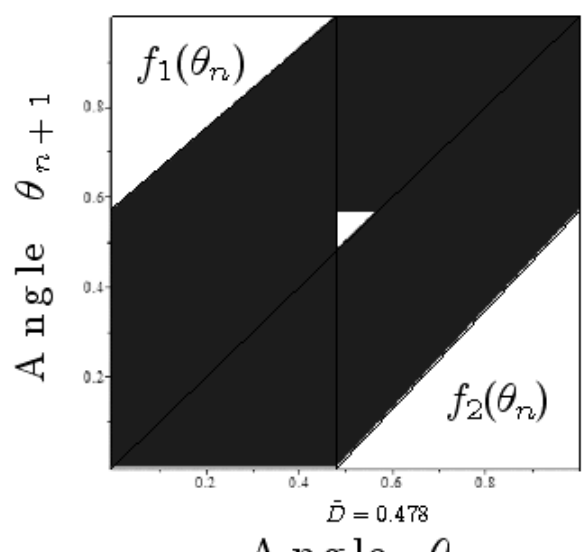

Angle $\theta_{n}$

(b)

Fig. 12: Quasiperiodic Motion in PCM $(\tilde{D}=0.478,(\mathbf{a}): n=1 \sim 100$, (b): $n=1 \sim 2100$ ) 


\section{$5 \quad$ Concluding Remarks}

Concluding remarks of this paper are summarized as follows;

(i) In the approximate PCM, there exist periodic motions which include almost periodic motions for the cutoff parameter $\tilde{D}>\tilde{D}_{\mathrm{C}}$, and there exist chaotic motions which include quasi-periodic motions $\tilde{\pi}_{r, s}(m, m+1)$ for the cut-off parameter $\tilde{D}<\tilde{D}_{\mathrm{C}}$.

(ii) In the approximate PCM, periodic motions which include almost periodic motions $\pi(m)$ and $\pi_{r, s}(m, m+$ 1) exist for positive integers $m \geq M_{\min }$.

(iii) The existence of chaotic motions in the PCM implies the existence of chaotic oscillations in the original nonlinear system of Van der Pol-Mathieu type.

(iv) From (i) (iii), it is seen that Lyapunov exponents of the PCM are effective for detecting chaotic oscillations in the original Van der Pol-Mathieu systems.

\section{References}

[1] R. May, Simple Mathematical Models with Very Complicated Dynamics, Nature, 261, 1976.

[2] H. Broer and F. Takens, Dynamical Systems and Chaos, Springer 2011.

[3] Y. Zheng, F. Wang and Y. Fu, Nonlinear Dynamic Stability of Moderately Thick Laminated Plates with Piezoelectric Layers, Int. J. of Nonlinear Sciences \& Numerical Simulation, 10 (4), pp.459-468, 2009.

[4] L. Xin, Modified Projective Synchronization of a New Hyper Chaotic System via Non Linear Control, Commun. Theor. Phys. 52, pp.274-278, 2009.

[5] L. Xin and C. Yong, General Projective Synchronization Between Rössler System and New Unified Chaotic System, Commun. Theor. Phys. 48, pp.132-136, 2007.

[6] J. Liu, J. Lu, Y. Shi, X. Li and Q. Tang, Different Type of Synchronization Phenomena in Unidirectional Coupled Unified Chaotic Systems, Int. J. of Innovative Computing, Information and Control 3, pp.697-708, 2007.

[7] B. Van der Pol, On Oscillation in a Triode Generator with Two Degrees of Freedom, Philos. Mag. 43, pp.700-719, 1927.

[8] M. Cartwright and J.E. Littlewood, On Nonlinear Differential Equation of the Second Order, J. London Math. Soc. 20, pp.180-189, 1945.

[9] E. M. EL-Abbasy, On the Periodic Solution the Van der Pol Oscillator with Large Damping, Proc R. Soc. Edinburgh Sect. A 100, pp.103-106, 1985.
[10] N. Levinson, A Second Order Differential with Singular Solutions, Ann. Math. 50, pp.127-153, 1949.

[11] U. Parlitz and W. Lauterborn, Period-Doubling and Devil's Staircases of the Driven Van der Pol Oscillator, Phys. Rev. A 36, pp.1428-1434, 1987.

[12] R. Mettin, U. Parlitz and W. Lauterborn, Bifurcation Structure of the Driven Van der Pol Oscillator, Int. J. Bifurcation Chaos 3, pp.1529-1555, 1993.

[13] F. Veerman and F. Verhulst, Quasi periodic phenomena in the Van der Pol-Mathieu equation Department of Mathematics, Utrecht University, pp.1-10, 2009.

[14] T. Yamaguchi and H. Ohtagaki, The Order of Appearance of Oscillation Modes of a Piecewise Linear Map, J. Phys. Soc. Jpn. 65, pp.3500-3512, 1996.

[15] T. Yamaguchi and H. Ohtagaki, Oscillation Modes of Forced Nonlinear Systems of Van der Pol Type, J. Phys. Soc. Jpn. 73, pp.588-598, 2004.

[16] A.J. Lichtenburg and M.A. Lieberman, Regular and Chaotic Dynamics, Springer, 1991.

[17] T. Yamaguchi and H. Ohtagaki Stochasticity of Nonlinear Systems of Van der Pol-Mathieu Type, Proc. of 42nd ISCIE Int. Symp. on Stochastic Systems Theory and Its Applications, pp.46-51, 2010. 